\title{
On the influence of junction structures on the mechanical and thermal properties of carbon honeycombs
}

\author{
Xiaokun Gu, ${ }^{1 *}$ Zhenqian Pang, ${ }^{2 *}$ Yujie Wei ${ }^{2 *}$ and Ronggui Yang ${ }^{3,4 *}$
}

\begin{abstract}
Carbon honeycomb is a 3-dimensional carbon allotrope experimentally discovered recently, but its lattice structure has not been well identified. In this paper, we perform density-functional theory (DFT) calculations to examine the stability of carbon honeycombs with different configurations (chirality and sidewall width). We find that graphene nanoribbons with both zigzag edges and armchair edges can form stable carbon honeycombs if $\mathrm{sp}^{3}$ carbon-carbon bonding is formed in the junction. We further study the mechanical properties and the thermal conductivity of carbon honeycombs with different chirality and the sidewall widths using both DFT calculations and molecular dynamics simulations. All these stable carbon honeycombs exhibit superior mechanical properties (large strength and ductility) and high thermal
\end{abstract}


conductivity (larger than $100 \mathrm{~W} / \mathrm{m} \mathrm{K}$ ) with a density as low as $0.5 \mathrm{~g} / \mathrm{cm}^{3}$. Light-weight carbon honeycombs could be promising functional materials for many engineering applications.

Keywords: carbon honeycomb, carbon allotrope, lightweight, thermal conductivity, mechanical strength, ductility 


\section{Introduction}

Lightweight materials with exceptional physical properties are highly desirable for many engineering applications. For example, lightweight materials with high mechanical strength can be utilized in spacecraft and skyscrapers, while those with high thermal conductivity can find their applications as heat sink materials for power electronics and high performance heat exchangers. In the past few decades, the research on various kinds of carbon allotropes, from bulk diamond and graphite to low-dimensional fullerene, carbon nanotube (CNT) and graphene, has stimulated remarkable progress in fundamental material sciences [1]. A few carbon allotropes, especially the low-dimensional graphene and CNT, possess high mechanical strength [2] and high thermal conductivity [3]. Using low-dimensional carbon materials as building blocks to construct three-dimensional (3-D) structures provides a promising routine to achieve multifunctional lightweight materials with strong strength and high thermal conductivity.

Very recently, carbon honeycombs, another 3-D carbon allotrope, has been experimentally synthesized by depositing vacuum-sublimated graphite [4], and immediately became a research focus [5-9]. Indeed long before the successful synthesis of carbon honeycombs, a few hypothesized structures, which were sometimes called carbon foams, had been proposed [10-17]. However, the stability of these hypothesized 3-D carbon structures is seldom examined. While most of these hypothesized 3-D structures can be regarded as armchair graphene nanoribbons connected through $\mathrm{sp}^{3}$ bonding $[12,14]$, our recent first-principles density functional theory (DFT) calculations has revealed that stable carbon honeycombs can also be formed by connecting zigzag graphene nanoribbons if $\mathrm{sp}^{3}$ carbon-carbon bonds are formed at the junction region [18]. Furthermore, DFT calculations and molecular dynamics simulations showed that the carbon honeycombs made of zigzag graphene nanoribbons possess appealing specific strength 
$\left(25 \mathrm{GPa} \cdot \mathrm{cm}^{3} / \mathrm{g}\right)$, which is much larger than any reported three-dimensional materials, and high thermal conductivity (>100 W/mK). These exceptional properties are due to the covalent nature of $\mathrm{C}-\mathrm{C}$ bonding in carbon honeycombs. The large specific strength and high thermal conductivity make carbon honeycombs excellent lightweight multifunctional materials for various applications.

Considering that carbon honeycombs can be made up of graphene nanoribbons with different chirality along their edges, it is hence of great relevance to explore how different structures in the junction of carbon honeycomb influence their stability, mechanical and thermal properties. In this paper, we perform DFT calculations to understand the stability and to predict mechanical strength of carbon honeycombs with different chirality and honeycomb sidewall (nanoribbon) width, and molecular dynamics simulations to study their thermal conductivity.

\section{Simulation method}

The essential step to realize a carbon honeycomb is to form a stable junction by connecting three graphene ribbons along their edges with appropriate bonding. How to connect them coherently is the key to maintain the extraordinary properties of graphene in 3-D carbon honeycombs. With a junction formed, nanoribbons with different widths can be connected to form carbon honeycombs with irregular hexagons. For simplicity, in this work we analyze only the carbon honeycombs with periodic structure along the junction, as shown in Figure 1(a). In the previous report [18], we investigated two types of junctions, one formed with the zigzag edges of graphene ribbons and the other along the armchair edges of graphene ribbons. The atomic details of the junctions and the graphene nanoribbons connected are presented in Figure 1(b-c). We termed the former as 5-5-8 junction since it is composed of an array of defective units, 
and each containing two 5-rings and one 8-ring, and the latter is called 6-6-6 junction as there are two coplanar 6-atom rings and one non-coplanar 6-atom ring in one period of the junction. Now we consider five carbon honeycombs which are constructed by the two kinds of junctions (5-5-8 and 6-6-6) and shifted defect arrangement along the two edges of a graphene nanoribbon, as shown in Figure 1(d-h). Three-dimensional views of these honeycombs are also presented in Supplementary Figure S1. In these structures, carbon atoms are in pink if they are in the junction lines, while other atoms are colored in cyan and silver colors. For brevity, we refer the five types of graphene nanoribbons studied in this paper, which are shown in Figure 1(d-h), as ZZ1, ZZ2, $\mathrm{ZZ3}, \mathrm{AC} 1$ and $\mathrm{AC} 2$ carbon honeycombs. Here the graphene nanoribbons of both $\mathrm{ZZ1}$ and $\mathrm{ZZ2}$ honeycombs only include odd number of hexagonal carbon rings and those of ZZ3 honeycombs have even number of hexagonal carbon rings. The difference between ZZ1 and ZZ2 carbon honeycombs is that the nanoribbons in ZZ1 honeycombs are asymmetric with respect to the center line of nanoribbons, while those in ZZ2 honeycombs are symmetric. For armchair-type of carbon honeycombs, the width of graphene nanoribbons in AC1 carbon honeycombs has to be roughly $n \sqrt{3} a_{0}$, where $n$ is an integral number and $a_{0}$ is carbon-carbon bond length, while that in AC2 carbon honeycomb is $\left(n+\frac{1}{2}\right) \sqrt{3} a_{0}$. 
(b)

(a)

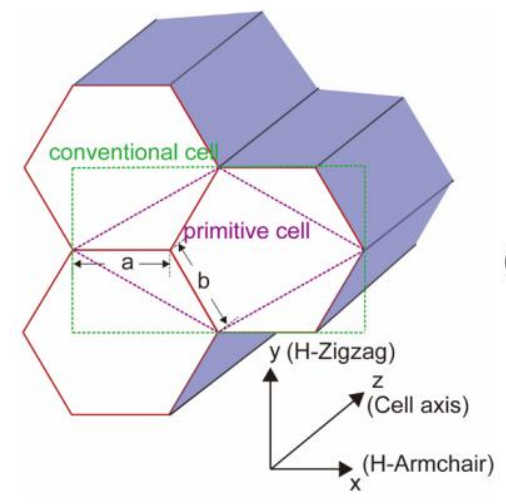

)

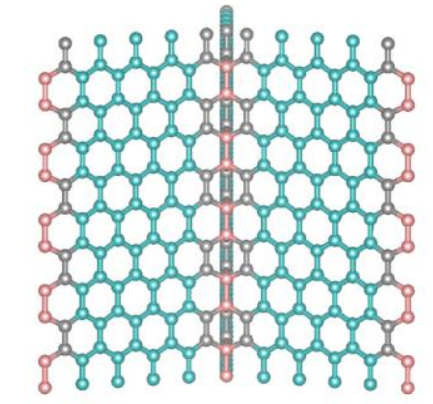

(d)

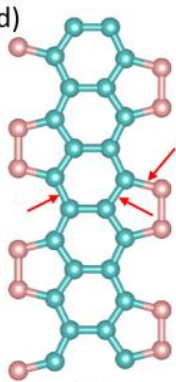

$\mathrm{ZZ1}$ (e)

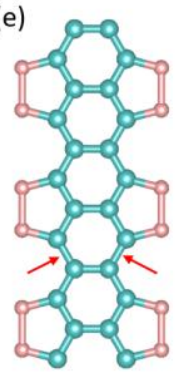

$\mathrm{ZZ2}$ (f)
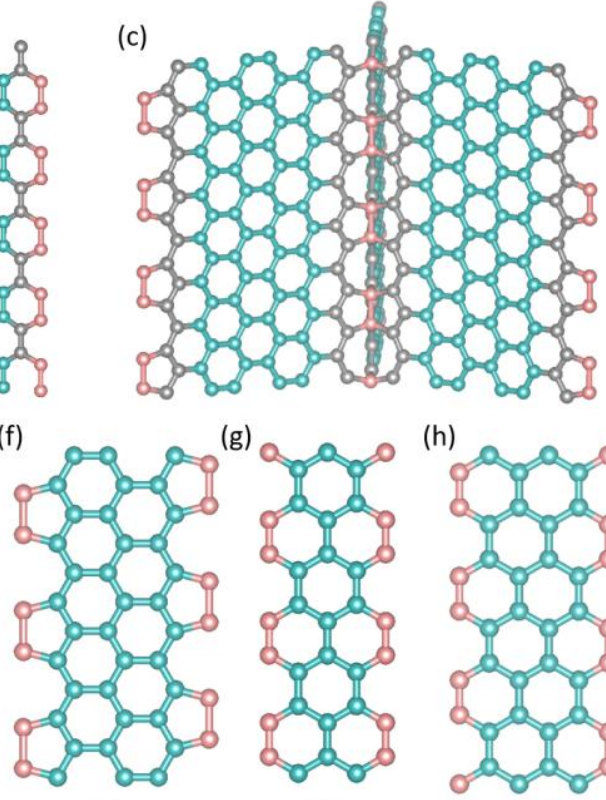

ZZ3

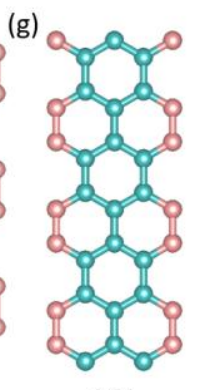

$\mathrm{AC1}$ (h)

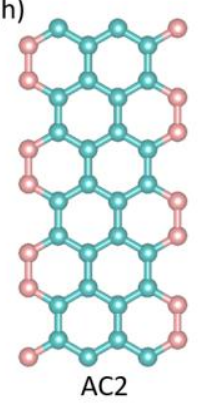

Figure 1. (a) Atomistic structure of 3-D carbon honeycombs. The green rectangle and violet parallelogram denote the conventional cell and the primitive cell of the 3-D carbon honeycomb structure, respectively. For clarity, only the face of the unit cell in the $x$-y plane is shown. (b) Local atomistic structure of the junction region of carbon honeycomb made up of armchair graphene nanoribbons. (c) Local atomistic structure of the junction region of carbon honeycomb made up of zigzag graphene nanoribbons. (d-h) five different types of nanoribbons that can be used to construct carbon honeycombs. The differences among these structures are discussed in main text. Carbon atoms are in pink if they are in the junction lines, while other atoms are colored in cyan and silver colors.

We perform DFT calculations using VASP package $[19,20]$ to examine the stability of the carbon honeycombs with different lattice structure including both chirality and sidewall widths and to study their mechanical properties. The DFT calculations are based on the projector augmented wave (PAW) pseudopotentials [21] and the generalized gradient approximation (GGA) of the Perdew-Burke-Ernzerhof (PBE) functional [22] with a kinetic-energy cut-off for the plane-wave basis set of $520 \mathrm{eV}$. To find the possible atomic structures of carbon honeycombs, 
we first construct the primitive unit cells (See Figure 1(a)) of different carbon honeycombs by connecting together the corresponding graphene nanoribbons, as shown in Figure 1(d-h), to form the honeycomb structure based on the equilibrium lattice parameter of graphene. The primitive unit cells of all the structures studied here are then relaxed in the DFT calculations using a conjugate gradient (CG) algorithm until the atomic forces are converged to $10^{-5} \mathrm{eV} / \AA$ and the total energy is minimized. To study the stability of these relaxed carbon honeycombs, we calculate their phonon dispersion curves using the Phonopy package [23], which is based on the supercell method. In the supercell method, the atoms in the supercell which contains several primitive unit cells of carbon honeycomb, are displaced by $\pm 0.01 \AA$ along the Cartesian directions, then the interatomic forces exerting on the atoms in the supercell are recorded. The second-order harmonic force constants are extracted through the finite difference scheme, so that the dynamical matrixes of the lattice are constructed with the extracted force constants and phonon dispersion relation is calculated by diagnosing the dynamics matrixes [24].

With the relaxed crystal structures of these carbon honeycombs, we then compute their strain-stress curves, from which the mechanical strength and failure strain can be determined, through DFT calculations. While the engineering stress $\sigma$, evaluated using the cross-section of the material before deformation, is frequently used to describe the mechanical state of materials, it would underestimate the true stress $\sigma^{\prime}$ for materials with large deformation, such as carbon honeycombs. Therefore, we report the true stress of carbon honeycombs in this work. To conveniently obtain the stress and strain along principal axes, we convert the primitive unit cells of the relaxed carbon honeycombs to the conventional ones, as illustrated by the green rectangle in Figure 1(a). In the following DFT calculations, we start from the unstrained honeycombs, and apply the quasi-static displacement-controlled deformation by imposing the deformation with a 
small strain increment. Once being stretched to a specific strain, the carbon honeycomb is then relaxed to achieve both zero stress along the directions perpendicular to the straining one and small atomic forces (less than $0.01 \mathrm{eV} / \AA$ ). As we calculate the stress based on the relaxed configuration of the simulation box, the obtained stress along the stretching direction is regarded as true stress. The results presented are calculated using the minimal conventional cells with periodic boundary conditions, and the calculated results are consistent with those using larger supercells, as demonstrated in Supplementary Figure S2, where an AC1 carbon honeycomb with a sidewall width of $5.2 \AA$ is examined as an example.

Many simulation techniques have been employed to study the thermal transport in solid crystals. While the first-principles-based Boltzmann transport equation approach have been successfully applied to predict the thermal conductivity of quite a few bulk $[25,26]$ and twodimensional crystalline materials [27-29], significant computational burden make it inapplicable for materials with complicated crystal structures, like carbon honeycombs. To avoid the computational challenges, we study the thermal conductivity of different stable carbon honeycombs using classical equilibrium molecular dynamics (MD) simulations, which are conducted via a well-tested software package LAMMPS [30]. The modified reactive empirical bond order (REBO) potential [31], whose parameters are optimized to predict the thermal conductivity of graphene and carbon nanotubes, are used to describe the interatomic interactions between carbon atoms. The thermal conductivity of graphene from our MD simulations is very close to that from the first-principles-based Boltzmann transport equation calculations where the quantum effects [29] are fully taken into account (within 15\%), as well as the measured data [24]. In the MD simulations, the position and velocity of each atom in the simulation system are determined by numerically integrating the Newton's law of motion of atoms using the velocity 
Verlet algorithm with a time step of 0.5 fs. The lattice thermal conductivity is then computed through the Green-Kubo linear-response formulation [32], which is expressed as

$$
\kappa^{\alpha \alpha}=\frac{1}{k_{B} T^{2} V} \int_{0}^{\infty}\left\langle J^{\alpha}(0) J^{\alpha}(t)\right\rangle d t
$$

where $V$ is the volume of the simulation domain, $T$ is the temperature, $J^{\alpha}(0) J^{\alpha}(t)$ is the heat current autocorrelation current auto-correlation function (HCACF) along the direction $\alpha$, and $<>$ denotes the ensemble average. The heat current, $J$, in Eq. (1) is derived based on the Hardy formula [33].

During the MD simulations we first thermalize the systems in isothermal-isobaric ensemble for 500 ps to control the temperature of the system and to maintain the pressure of the system to zero by changing the size of the simulation domain. Then, we run the simulation under the canonical ensemble for another 500 ps to further relax the system. After that, the simulation system is switched to micro-canonical ensemble for $10 \mathrm{~ns}$, during which the heat flux information is collected for calculating the thermal conductivity. We repeat our simulations more than 10 times for each temperature to reduce the statistical error. In each independent simulation run, the initial conditions (initial atom velocities) are set differently. The averaged HCACF from each independent run, as shown in Figure 2, is found to decay to zero within 250 ps, which is thus chosen as the upper bound of the integral in Eq. (1). The thermal conductivity is obtained by integrating the averaged $\mathrm{HCACF}$, and the error bar of the calculated thermal conductivity is determined as the standard error of the calculated thermal conductivities from these independent runs. 


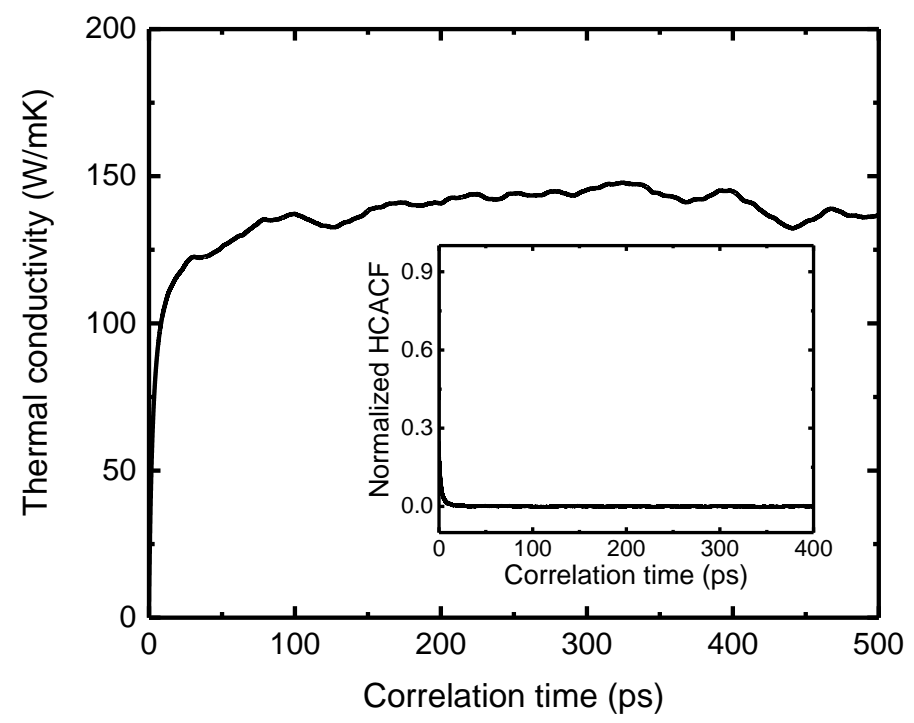

Figure 2. The calculated thermal conductivity of an ACl carbon honeycomb with the sidewall width of $5.2 \AA$ obtained by the Green-Kubo relation plotted against the correlation time. The insets show the decay of normalized heat current autocorrelation function (HCACF).

\section{Results and discussion}

\subsection{Phonon dispersion and stability}

As the cohesive energy is the energy required to break the chemical bonds in a material, the magnitude of cohesive energy can serve as a rough indicator for the stability of a material among different phases. Figure 3 shows the calculated cohesive energy per atom in the carbon honeycombs with different sidewall widths and chirality. The cohesive energy per atom of each type of carbon honeycombs decreases with the increase of the sidewall width, since the cohesive energy per atom should be approaching to that of graphene when the sidewall width further increases. By comparing the five types of carbon honeycombs studies in this work, we find that generally the $\mathrm{AC} 1$ and $\mathrm{AC} 2$ carbon honeycombs have lower cohesive energy than the three zigzag-type carbon honeycombs with similar sidewall width. This indicates that the synthesized 
carbon honeycombs are more likely to be armchair type since they are energetically more favorable. As the increase of the width of nanoribbons, the difference in cohesive energy among these honeycombs becomes smaller.

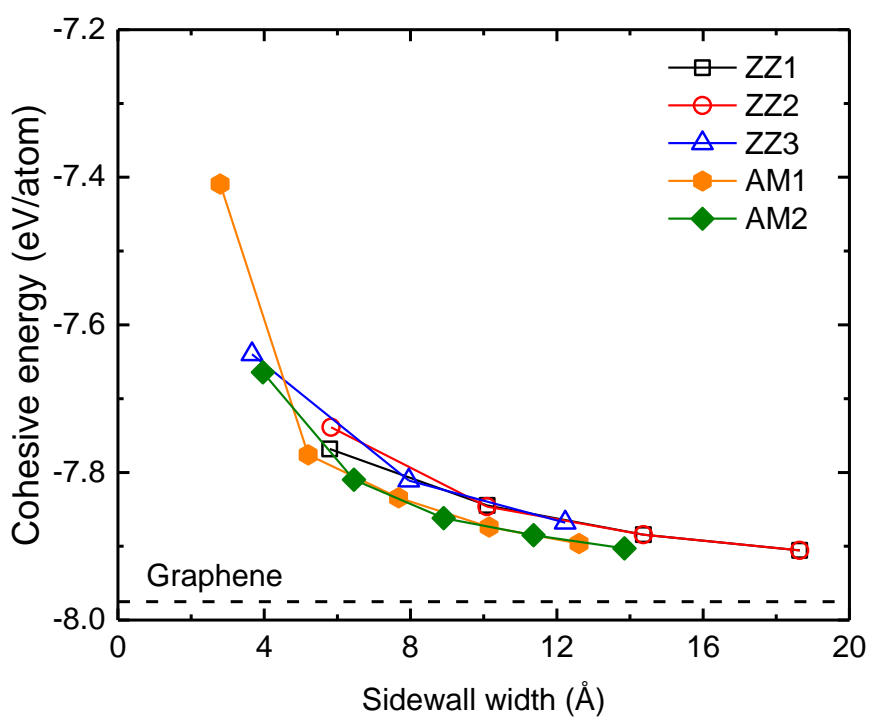

Figure 3. The calculated cohesive energy of carbon honeycombs with different chirality and sidewall widths.

While the cohesive energy is a measure of the relative stability of different structures, it does not necessarily mean that the structure with a higher cohesive energy cannot exist. For example, both graphite and diamond exist in nature although graphite is slightly more energetically stable than diamond [31]. The key to the stability of a structure is indeed that there exists sufficiently high energy barrier (to the unstable state) from a local energy minimum to resist thermal perturbation, which can be inferred by the non-negative phonon frequencies. Therefore, to further examine the stability of these carbon honeycombs, we calculate their phonon dispersion relations as shown in Figure 4. Due to the computational limitation, we only examine the carbon 
honeycombs with sidewall width smaller than $6 \AA$. All of the carbon honeycombs with zigzagtype side walls are found to have positive phonon frequencies (see Figure $4(\mathrm{a}-\mathrm{c})$ ), if $\mathrm{sp}^{3}$ bonding occurs at the junction regions. For carbon honeycombs with armchair-type side walls, the structures are also stable when the widths of the side walls are $5.2 \AA$ and $4.0 \AA$ (see Figure $4(\mathrm{~d}$ e)). However, if the width becomes smaller, the structure is no longer dynamically stable. As seen in Figure 4(f), negative phonon frequencies can be clearly identified in phonon dispersion curves for the AC1 carbon honeycomb with a side wall width of $2.8 \AA$. By examining the detailed atomic structures of the carbon honeycomb, as presented in Figure 5, we can observe that the tetrahedral angles deviate significantly from the ideal $109.5^{\circ}$ to $103.3^{\circ}$ and $115.1^{\circ}$. The large deformation might cause the $\mathrm{AC} 1$ carbon honeycomb with small sidewall width transitions to other more stable configuration. We note that although the phonon dispersion relations of carbon honeycombs with sidewall width larger than $6 \AA$ are not examined in this work, the larger carbon honeycombs are expected to be stable as the degree of the deformation in the junction regions tends to be smaller. 
(a)
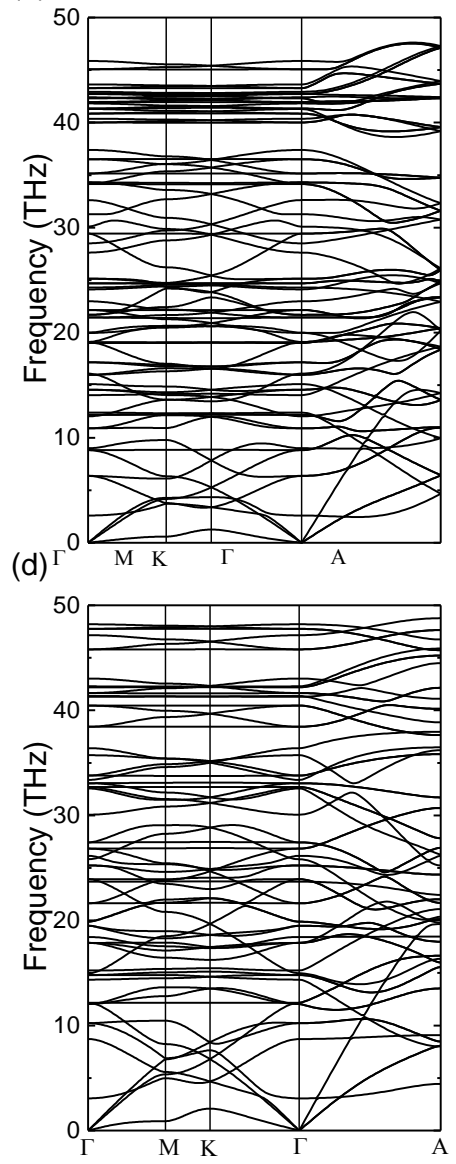

(b)
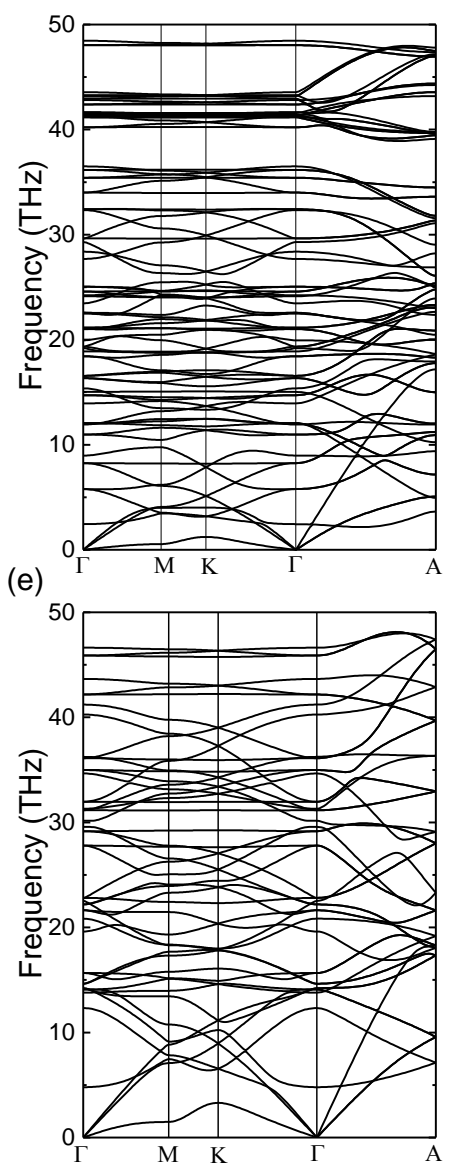

(c)

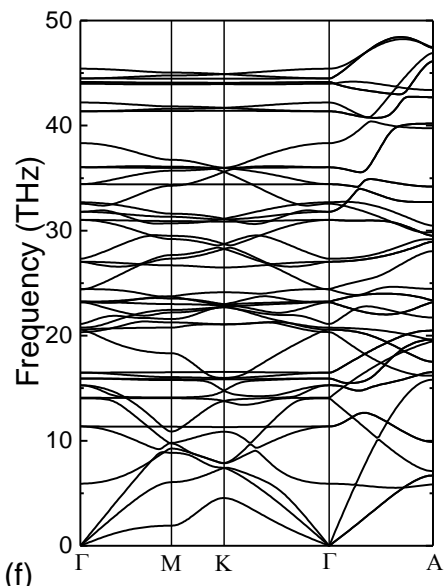

(f)

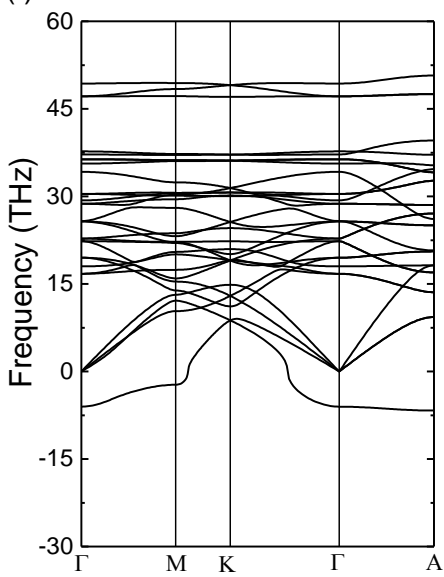

Figure 4. The calculated phonon dispersion of carbon honeycombs. (a) ZZ1 with a sidewall width of $5.8 \AA$; (b) ZZ2 with a sidewall width of $5.8 \AA$; (c) ZZ3 with a sidewall width of $3.7 \AA$; (d) AC1 with a sidewall width of $5.2 \AA$; (e) AC2 with a sidewall width of $4.0 \AA$; (f) AC1 with a sidewall width of $2.8 \AA$. 

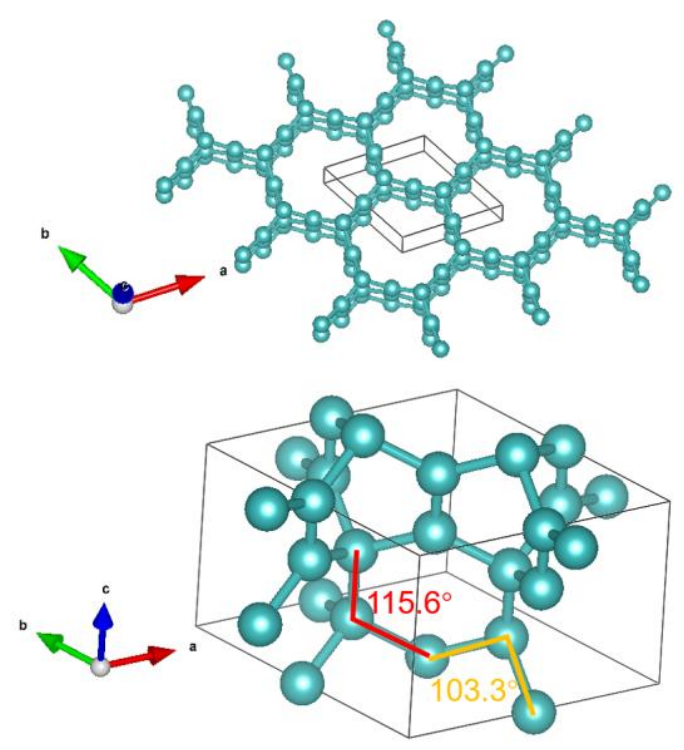

Figure 5. The atomic structure of ACl carbon honeycombs with sidewall width of $2.8 \AA$.

\subsection{Mechanical properties}

We first calculate mechanical properties along the cell-axis direction for the five types of honeycombs with different sidewall widths. The strain-stress curves are shown in Figure 6(a-e). Overall, these carbon honeycombs have much better strength and ductility compared with traditional metals such as aluminum and stainless steels $[34,35]$. As the sidewall width increases, the strength and ductility gradually decreases. The sidewall width-dependent mechanical properties can be understood from the fact that the carbon honeycombs with smaller sidewall widths have denser array of graphene nanoribbons to sustain the mechanical loading.

To further reveal the relationship between the mechanical properties with the sidewall widths of carbon honeycombs, we define effective stress through the ratio between the tension and effective cross-section area of the honeycombs (the area occupied by graphene nanoribbons), which is expressed as 


$$
\sigma_{e f f}=\beta \frac{\sqrt{3} \sigma \prime a}{3 t}
$$

where $\sigma^{\prime}$ is true stress calculated, $a$ and $t$ are the length and thickness of graphene nanoribbon sidewall, respectively. The choice of $t$ is controversial in literature [36-38], but it only serves a scaling factor in Eq. (2). Here, we use $t=3.4 \AA$, a thickness derived from bulk graphite[39]. $\beta$ is a correction factor used to take into account the Poisson effect. The effective stress, Eq. (2), is derived under the assumption that the deformation of carbon honeycombs along the armchair and zigzag directions of the honeycomb structure, which are denoted as $\mathrm{H}$-Armchair direction and $\mathrm{H}$ Zigzag direction, are all caused by the shortening of the sidewall width when the tension is applied along cell-axis direction. As the Poisson's ratio of graphene with small deformation is $v=-0.6 \varepsilon+0.17$ and $v=-0.34 \varepsilon+0.17$ if the graphene is stretched along the zigzag and armchair direction [40], respectively, $\beta$ is expressed as

$$
\begin{aligned}
& \text { Tension along H-Armchair direction: } \beta=0.6 \varepsilon^{2}-0.17 \varepsilon+1 \quad(\varepsilon<0.3), \\
& \text { Tension along H-Zigzag direction: } \beta=0.34 \varepsilon^{2}-0.17 \varepsilon+1 \quad(\varepsilon<0.3),
\end{aligned}
$$

where $\varepsilon$ is the strain applied on the carbon honeycomb. The strain-effective stress curves are shown in Figure 6(f-k). These curves of the same type of carbon honeycombs with different sidewall width almost overlap with each other except those carbon honeycombs with nanoribbon sidewall width smaller than $10 \AA$. This indicates that the strain-stress curve of honeycomb is dominantly determined by the deformation of graphene nanoribbons when the sidewall width is larger than $10 \AA$, and the honeycombs inherit good mechanical properties of graphene nanoribbons. This observation agrees well with the continuum mechanics theory [41] and the Saint-Venant's principle [42], as the effects of the detailed atomic structure near the junctions becomes negligible. Based on the obtained relationship between the effective stress and strain of each type of carbon honeycombs, it is possible to deduce the mechanical properties of 
honeycombs with larger sizes that are not calculated in this work due to the computational limitation. By comparing the results of the zigzag carbon honeycombs with the armchair carbon honeycombs of a similar sidewall width, the strengths of the zigzag carbon honeycombs are found to be slightly larger than the armchair ones, which is consistent with the fact that the strength of graphene is larger when the tension is applied along the zigzag direction of graphene [40].
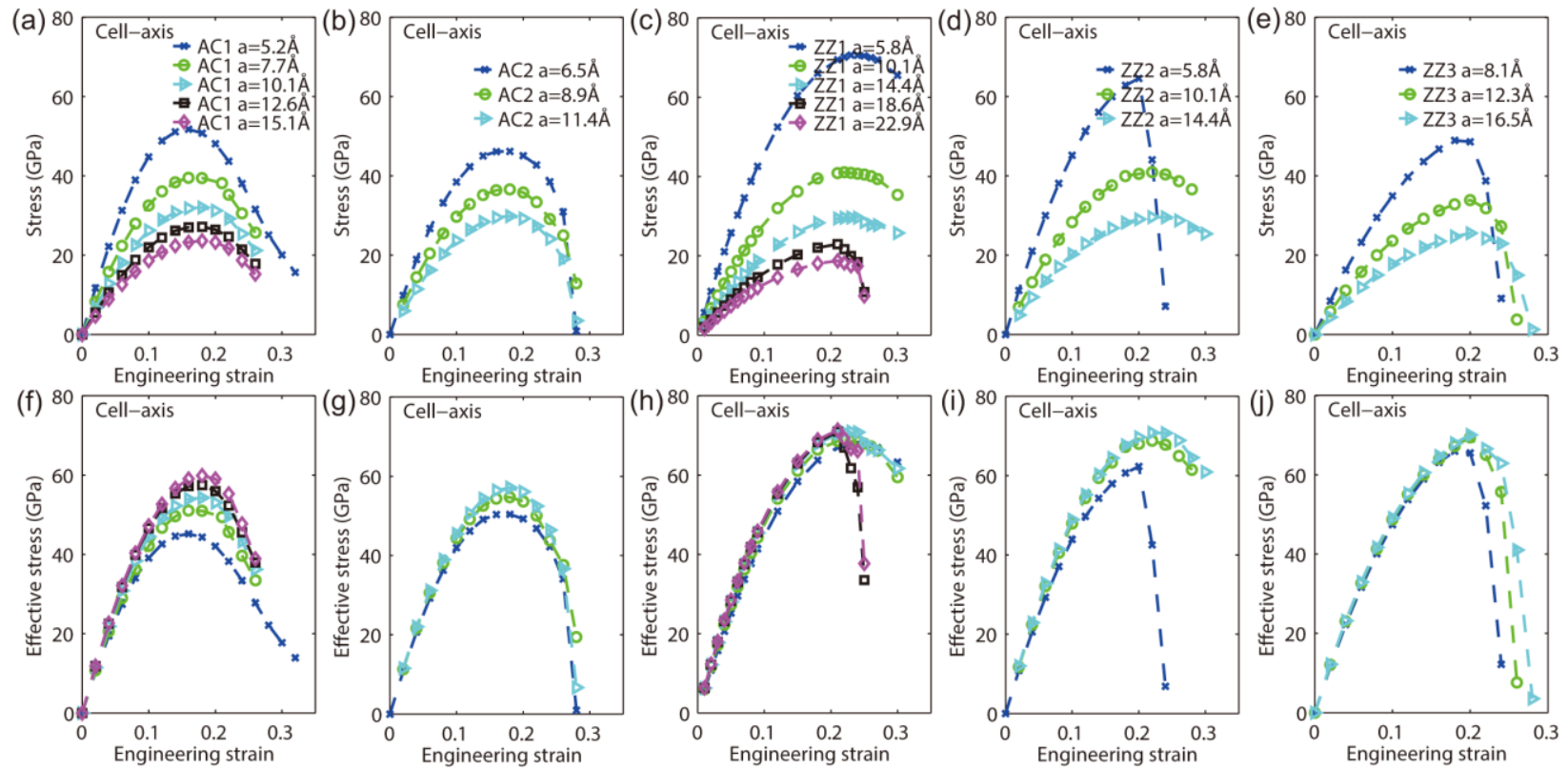

Figure 6. (a-e) The calculated stress-strain curves for AC1, AC2, ZZ1, ZZ2, ZZ3 carbon honeycombs with different sidewall widths when the tension is applied along the cell-axis direction. $(f-j)$ The corresponding effective stress-strain curves.

Although the effective stress-strain relation of carbon honeycombs is mostly determined by the intrinsic properties of graphene nanoribbons when the sidewall width is large, the atomic structure of different graphene nanoribbons can cause some difference in mechanical behavior of carbon honeycombs with small sidewall width. For example, both the strength and the failure strain of ZZ2 carbon honeycomb with a sidewall width of $5.8 \AA$ are slightly smaller than those of 
the ZZ1 one. This difference can be explained by the symmetry of nanoribbons. Due to the symmetric structure, the narrowest part of nanoribbons of ZZ2 carbon honeycomb is narrower than that of ZZ2 carbon honeycomb. As a result, the effect of stress is overcome only by the elongation of two C-C bonds (as indicated by arrows in Figure 1(e)) near the narrowest part of ZZ2 carbon honeycomb, but the stress-induced deformation is shared by three C-C bonds (as indicated by arrows in Figure 1(d)). When the same stress is applied, C-C bonds in than ZZ2 carbon honeycomb is more elongated than in ZZ1 carbon honeycomb, and more likely to be broken. Therefore, symmetry of junction reduces the strength of carbon honeycombs with small sidewall width. As the increase of sidewall width, the influence of the number of bonds on the strength weakens and become secondary. This conclusion is also true for the armchair carbon honeycombs, as the strength of AC2 honeycomb with a $6.5 \AA$ sidewall width is even larger than that of AC1 honeycomb with a $5.2 \AA$ sidewall width.

We also study the mechanical behavior of carbon honeycombs when the strain is applied along the other two directions, i.e., the armchair- and zigzag-directions of the honeycomb structure, as shown in Figure 7. When we stretch carbon honeycombs along the armchair direction, we find that the mechanical strength and failure strain decrease with the increase of sidewall width (see Figure 7(a-e)). From microscopic point of view, a given stress could lead to larger bending moment about the junction when the sidewall width is larger. Assuming that the failure bending moment is not dependent on the sidewall width, larger sidewall would result in smaller strength and smaller failure strain. These five types of carbon honeycombs with the similar sidewall width have very similar stress-strain curves, but the stress under the same strain of the zigzag structures are slightly smaller than that of the armchair ones. To understand such a difference, we analyze the deformed AC1 and ZZ1 carbon honeycombs with $24 \%$ strain along 
H-Armchair direction, as shown in Figure 8. It is found that the rotation angle of the armchair structure is much larger than the zigzag one, indicating that the junction bonds in the armchair carbon honeycomb has greater resistance to the rotational deformation in contrast to those in zigzag ones. Figures $8(\mathrm{~b}-\mathrm{c})$ shows the charge density of different junctions at $24 \%$ strain. We use $\mathrm{AB}$ and $\mathrm{BC}$ to refer two directions along the nanoribbon sheets, as shown in Figure 8(a). Compared with the charge density between junction atom with sidewall atoms (dash line box), we find that the charge density along the $\mathrm{AB}$ direction is lower than that along the $\mathrm{BC}$ direction in two structures. In Figure 7(f-j), we show stress-strain curves when the strain is applied along the H-Zigzag direction. Similar to the case of tension along the H-Armchair direction, the mechanical strength and failure strain decrease with the increase of sidewall width.

Apart from the strain-stress curves, the Young's modulus is of particular importance to quantify the mechanical response of a material under small deformations. According to continuum mechanics of cellular solids [43], we can deduced the Young' modulus of these honeycombs in three directions as: $E_{H-\text { Armchair }}=E_{H-\text { Zigzag }}=\frac{4 \sqrt{3}}{3}\left(\frac{t}{a}\right)^{3} E^{*}$ and $E_{\text {Cell-axis }}=$ $\frac{4 \sqrt{3}}{3}\left(\frac{t}{a}\right) E^{*}$, respectively, where $E^{*}$ means the Young's modulus of the side wall. As the sidewall width increases, the Young' modulus decreases accordingly. From the stress-strain curves in Supplementary Figure S3, we can find the trend of the Young's modulus fit well with classical continuum mechanics of cellular solids, regardless of the type of junctions. 

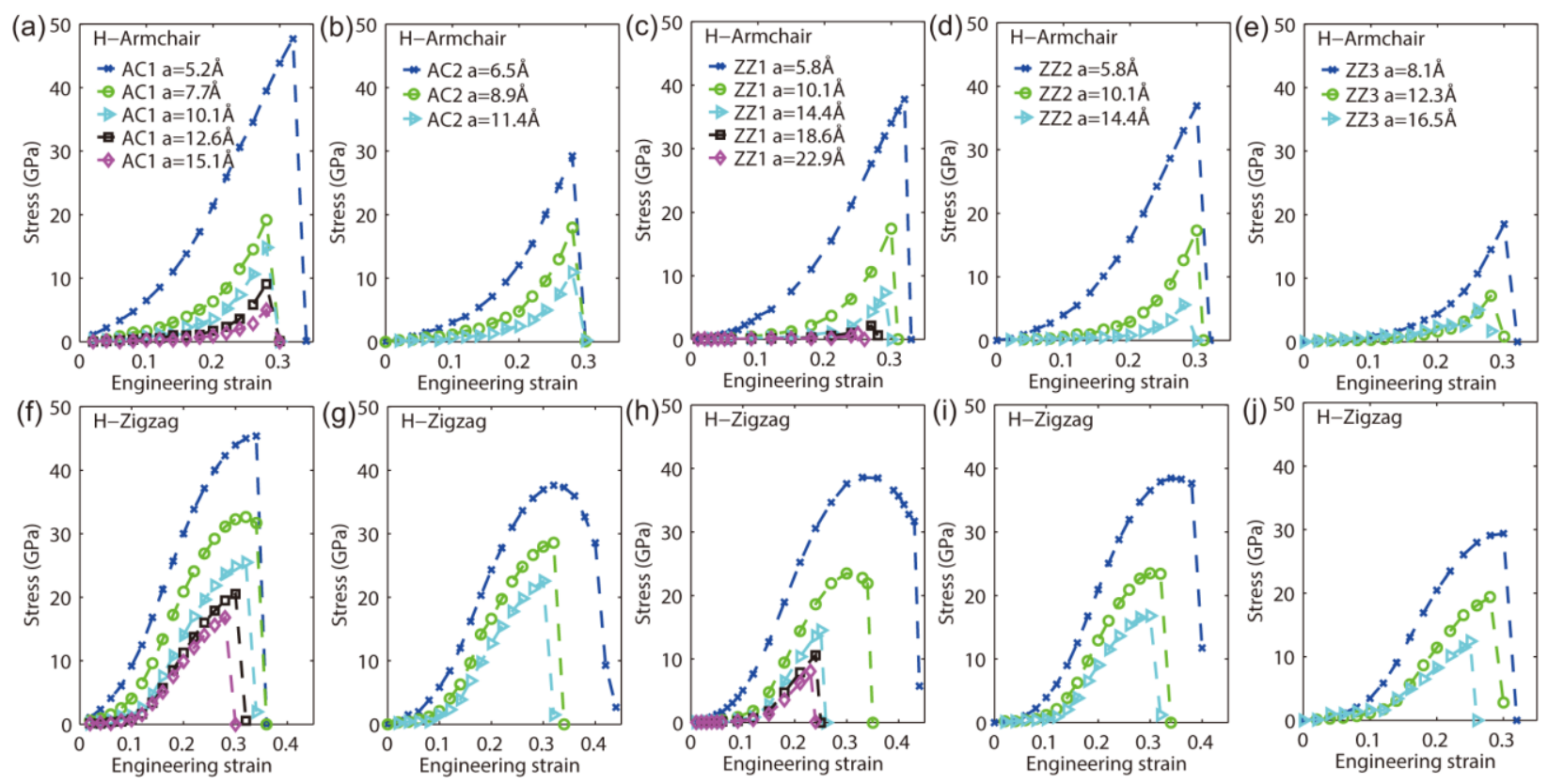

Figure 7. (a-e) The calculated stress-strain curves for AC1, AC2, ZZ1, ZZ2, ZZ3 carbon honeycombs with different sidewall widths when tension is applied along the armchair direction of the honeycomb structures. (f-j) The stress-strain curves when tension is applied along the zigzag direction of the honeycomb structures.

(a) armchair structure (24\% strain)

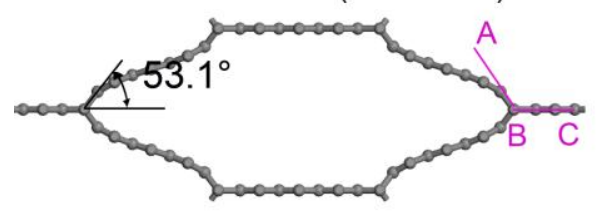

zigzag structure ( $24 \%$ strain)

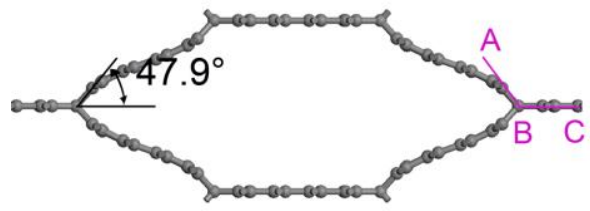

(b)

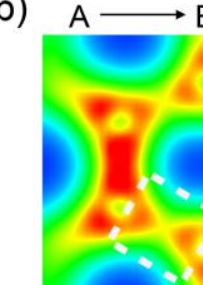

$\mathrm{B} \longrightarrow \mathrm{C}$

(c)

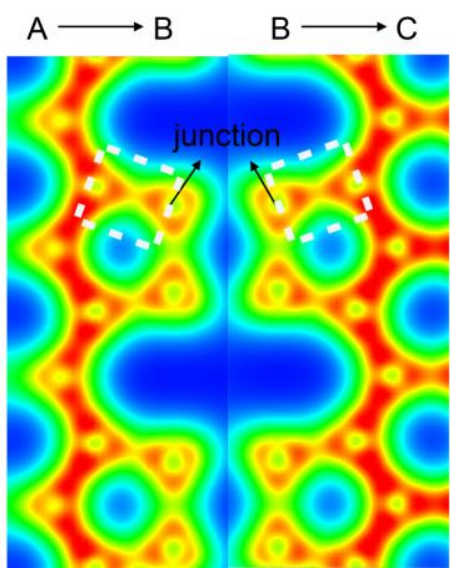

Figure 8. (a) The deformation of ACI and ZZ1 carbon honeycomb with the same sidewall size (10.1 $)$ when tensile is applied along the H-Armchair direction. Charge density of two structure with 24\% strain in junction: (b) AC carbon honeycomb and (c) ZZ carbon honeycomb. The region of dash line box represents the charge density of junction atom and sidewall atom. 


\subsection{Thermal properties}

Equilibrium molecular dynamics simulations were conducted to study the thermal conductivity of carbon honeycombs. Since the calculated thermal conductivity from equilibrium molecular dynamics simulations could be sensitive to the size of simulation domains, we carefully test the dependence of thermal conductivity on the size of simulation domain using the carbon honeycombs made of ZZ1 and AC1 graphene nanoribbons as shown in Figure 1(d) and Figure $1(\mathrm{~g})$, with sidewall widths of $5.2 \AA$ and $5.2 \AA$ as examples. The calculated thermal conductivities of carbon honeycombs using different simulation domain size are summarized in Supplementary Table S1. It is found that the calculated thermal conductivity is roughly unchanged when the size of simulation domain is larger than $4 \times 4 \times 6(4 \times 4 \times 12)$ unit cells for carbon honeycomb with zigzag (armchair) nanoribbbons. Therefore, a simulation domain made up of $4 \times 4 \times 6(4 \times 4 \times 12)$ primitive unit cells are employed to calculate the thermal conductivity of carbon honeycombs with zigzag (armchair) nanoribbbons, which are presented in the rest of this paper.

Figure 9 shows the thermal conductivities along the cell axis of the five types of carbon honeycombs with different sidewall widths at room temperature. The thermal conductivity values are found to be sensitive to the sidewall width. As the sidewall width increases from 0.5 $\mathrm{nm}$ to $2 \mathrm{~nm}$, the thermal conductivity of all types of carbon honeycombs decreases. The thermal conductivity is around $200 \mathrm{~W} / \mathrm{mK}$ when the sidewall width is about 4-5 $\AA$, which is comparable to most highly conductive metals and a few high thermal conductivity semiconductors such as $\mathrm{GaN}$ and silicon. The thermal conductivity reduction with respect to the sidewall width can be easily understood by the fact that larger sidewall width results in the larger porosity or smaller filling ratio of graphene nanoribbons in the carbon honeycombs. The density of carbon honeycombs with sidewall width from $0.4 \mathrm{~nm}$ to $1.5 \mathrm{~nm}$ reduces from $1.8 \mathrm{~g} / \mathrm{cm}^{3}$ to $0.5 \mathrm{~g} / \mathrm{cm}^{3}$, 
taking $\mathrm{AC} 1$ and $\mathrm{AC} 2$ carbon honeycombs as examples. Lower density indicates lower heat capacity and thus the lower thermal conductivity since the lattice thermal conductivity of a crystalline material is determined by $K \sim C v^{2} \tau$, with heat capacity $C$, phonon group velocity $v$ and phonon relaxation time $\tau$, from simple phonon kinetic theory. If we normalize the thermal conductivity of carbon honeycombs with the density, the normalized value increase with density, as shown in Figure 10, where AC1 and AC2 carbon honeycombs are used as examples. Such an increase of the density-normalized thermal conductivity can be qualitatively linked to the widthdependent thermal conductivity of graphene nanoribbon, where the thermal conductivity generally increases with the nanoribbon width due to less phonon-boundary scattering. Because the normalized thermal conductivity keeps increasing when the sidewall width decreases, the thermal conductivity of carbon honeycombs should decease slower than the drop of density. This explains that carbon honeycombs even with a density of $0.55 \mathrm{~g} / \mathrm{cm}^{3}$ (the sidewall width is around $1.5 \mathrm{~nm}$ ) can maintain high thermal conductivity (above $100 \mathrm{~W} / \mathrm{mK}$ ).
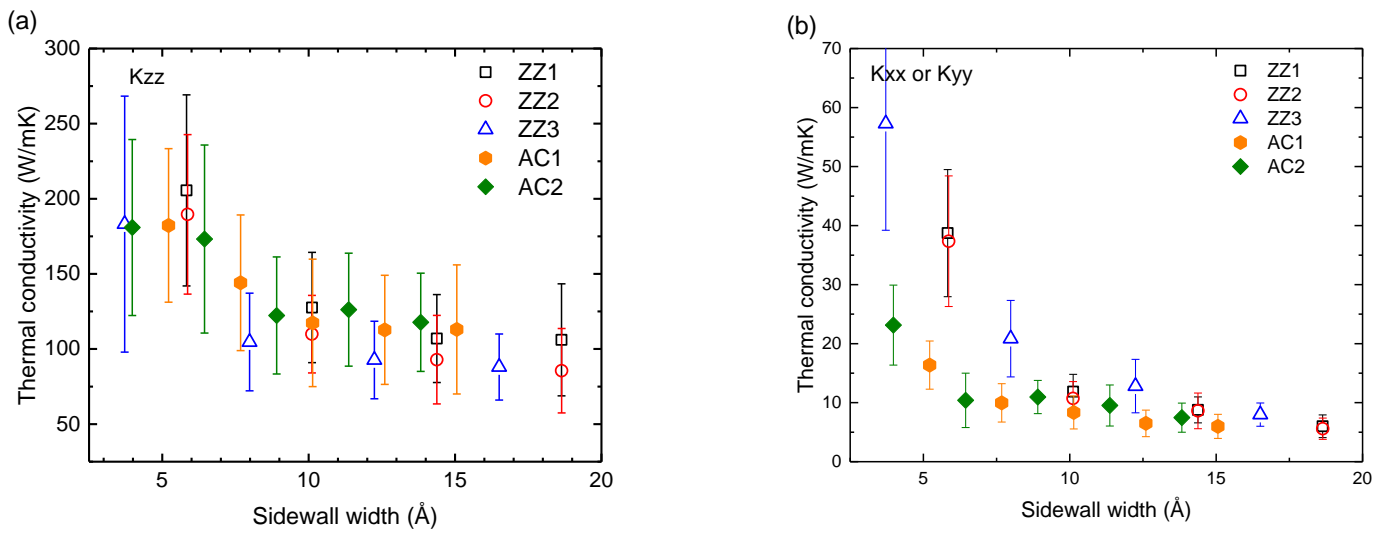

Figure 9. The calculated thermal conductivity of carbon honeycombs as a function of sidewall width (a) along the cell-axis direction, (b) along h-zigzag (h-armchair). 
The chirality of graphene nanoribbons of carbon honeycombs might influence the thermal conductivity of carbon honeycombs. Studies on the chirality-dependent thermal conductivity of graphene nanoribbons using molecular dynamics simulation has been well documented in literature [24]. It was shown that for the same width graphene nanoribbons, the one with zigzag edge usually have higher thermal conductivity than that with the armchair edge. Many explanations have been proposed to interpret the chirality-dependent thermal conductivity for graphene nanoribbons, such as the difference on the strength of phonon-boundary scattering [44, 45], phonon group velocity [46] or the degrees of localization [47]. Considering that the armchair graphene edge has more defects per unit length than the zigzag edge, it is very likely phonons in the armchair graphene nanoribbon scatter more often with the boundary which results in its lower thermal conductivity. However, we do not observe obvious difference on the thermal conductivity of carbon honeycombs made of different types of graphene nanoribbons, which is within error bars from molecular dynamics simulations, except the ZZ3 carbon honeycombs, which are of lower thermal conductivity than all other types studied. The weak dependence of thermal conductivity on the chirality could be understood from that the edges of all types of nanoribbons in honeycomb structure are similar, which can be treated as an array of atom pairs, as shown in Figure 1. Therefore, compared with graphene nanoribbons, the chirality-dependence in carbon honeycomb should be weaker. 


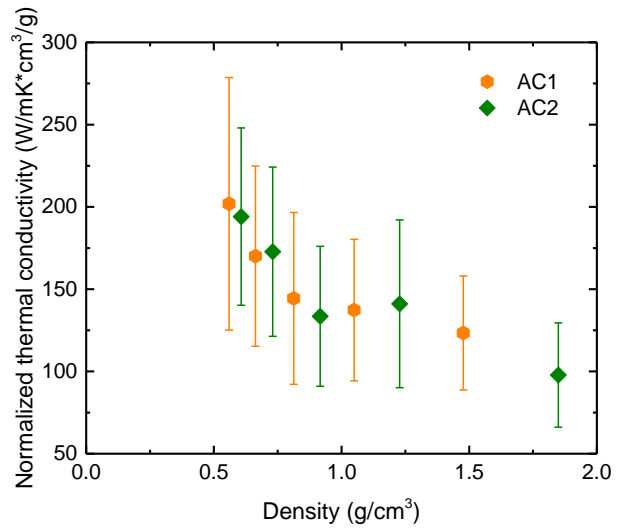

Figure 10. The density-normalized thermal conductivity of carbon honeycombs along the cellaxis direction as a function of density.

In Figure 9(b), we show the thermal conductivity of carbon honeycomb along h-zigzag (harmchair) direction. The thermal conductivity is about one order of magnitude smaller than that along cell axis direction. Interestingly, unlike the observation that the thermal conductivity along the cell axis direction is insensitive to the chirality, the in-plane thermal conductivity of armchair-type carbon honeycomb is only about half of that in the zigzag-type ones when the sidewall width is smaller than $7 \AA$. The difference becomes significant only when the sidewall contains less than 4 atomic planes. It ought to be governed by local phonon transport in the junction. Sidewalls in either zigzag- or armchair-type carbon honeycomb are graphene nanoribbons with high length-to-width aspect ratio. With increase sidewall width, the thermal conductivity of graphene nanoribbon approaches that of graphene sheet, which is isotropic. As a result, the thermal conductivity of carbon honeycomb is no longer affected by the chirality when the sidewall length is larger than $15 \AA$. 


\section{Conclusions}

In summary, we study the stability, the mechanical properties and the thermal conductivity of carbon honeycombs with different chirality and sidewall widths using DFT calculations and equilibrium molecular dynamics simulations. Carbon honeycombs with both zigzag and armchair graphene nanoribbons are stable if the sidewall width is larger than $3 \AA$, as their phonon frequencies are all non-negative. The carbon honeycombs inherit good mechanical properties of graphene nanoribbons, such as large strength and ductility. The strengths of carbon honeycombs made of zigzag graphene nanoribbons are found to be slightly larger than the armchair ones, due to the anisotropic response of graphene under tension. The strain-stress curve of honeycomb is found to be dominantly determined by the deformation of graphene nanoribbons when the width is larger than $10 \AA$. The thermal conductivity of carbon honeycombs along the cell axis is found to be larger than $100 \mathrm{~W} / \mathrm{mK}$ even when the density is only $0.5 \mathrm{~g} / \mathrm{cm}^{3}$. The effect of chirality of graphene nanoribbons on thermal conductivity is not significant, since the edges of all types of nanoribbons in honeycomb structure is similar.

The carbon honeycombs studied in this work are of idealized uniform crystal structures, but the synthesized carbon honeycombs are likely to be irregular honeycomb structures. The thermal conductivity of these carbon honeycombs might be different from the idealized ones. To estimate the thermal conductivity, more complicated composite model should be employed, which is worthy of further investigation. In addition, defects, vacancies, and functional groups, might occur on the graphene wall, which might alter the mechanical and thermal properties to some extent. Our simulations could serve as a benchmark for future studies on more realistic carbon honeycombs. 


\section{Acknowledgements}

RY would like to acknowledge the support by the National Science Foundation (Award No. 1512776) along with the Teets Family Endowed Doctoral Fellowship from CU Engineering awarded to XG. YW acknowledges the support from National Natural Science Foundation of China (NSFC) (11425211). The calculations are performed at both the Janus supercomputer, which is supported by the National Science Foundation (Grant No. 0821794), and the Supercomputing Center of CAS. The authors acknowledge the insightful discussions with high school student intern Miss. Yunfan Yang.

\section{References:}

[1] Meunier V, Souza Filho A, Barros E, Dresselhaus M. Physical properties of low-dimensional s p 2-based carbon nanostructures. Reviews of Modern Physics. 2016;88(2):025005.

[2] Lee C, Wei X, Kysar JW, Hone J. Measurement of the elastic properties and intrinsic strength of monolayer graphene. science. 2008;321(5887):385-8.

[3] Balandin AA. Thermal properties of graphene and nanostructured carbon materials. Nature materials. 2011;10(8):569-81.

[4] Krainyukova NV, Zubarev EN. Carbon Honeycomb High Capacity Storage for Gaseous and Liquid Species. Physical review letters. 2016;116(5):055501.

[5] Zhong C, Chen Y, Xie Y, Yang SA, Cohen ML, Zhang S. Towards three-dimensional Weylsurface semimetals in graphene networks. Nanoscale. 2016;8(13):7232-9.

[6] Yang Z, Lan G, Ouyang B, Xu L-C, Liu R, Liu X, et al. The thermoelectric performance of bulk three-dimensional graphene. Materials Chemistry and Physics. 2016;183:6-10. 
[7] Zhang Z, Kutana A, Yang Y, Krainyukova NV, Penev ES, Yakobson BI. Nanomechanics of carbon honeycomb cellular structures. Carbon. 2017;113:26-32.

[8] Wei Z, Yang F, Bi K, Yang J, Chen Y. Thermal transport properties of all-sp 2 threedimensional graphene: Anisotropy, size and pressure effects. Carbon. 2017;113:212-8.

[9] Chen Y-P, Zhong C, Zhang Z, Xie Y, Zhang S. Electron and phonon properties and gas storage in carbon honeycomb. Nanoscale. 2016.

[10] Liu AY, Cohen ML, Hass K, Tamor M. Structural properties of a three-dimensional allsp 2 phase of carbon. Physical Review B. 1991;43(8):6742.

[11] Karfunkel HR, Dressler T. New hypothetical carbon allotropes of remarkable stability estimated by MNDO solid-state SCF computations. Journal of the American Chemical Society. 1992;114(7):2285-8.

[12] Park N, Ihm J. Electronic structure and mechanical stability of the graphitic honeycomb lattice. Physical Review B. 2000;62(11):7614.

[13] Umemoto K, Saito S, Berber S, Tománek D. Carbon foam: Spanning the phase space between graphite and diamond. Physical Review B. 2001;64(19):193409.

[14] Kuc A, Seifert G. Hexagon-preserving carbon foams: Properties of hypothetical carbon allotropes. Physical Review B. 2006;74(21):214104.

[15] Zhu Z, Tománek D. Formation and stability of cellular carbon foam structures: an ab initio study. Physical review letters. 2012;109(13):135501.

[16] Zhu Z, Fthenakis ZG, Guan J, Tománek D. Topologically protected conduction state at carbon foam surfaces: an Ab initio Study. Physical review letters. 2014;112(2):026803. 
[17] Kawai T, Okada S, Miyamoto Y, Oshiyama A. Carbon three-dimensional architecture formed by intersectional collision of graphene patches. Physical Review B. 2005;72(3):035428.

[18] Pang Z, Gu X, Wei Y, Yang R, Dresselhaus MS. Bottom-up design of three-dimensional carbon-honeycomb with superb specific strength and high thermal conductivity. Nano Letters. 2017.

[19] Kresse G, Furthmüller J. Efficiency of ab-initio total energy calculations for metals and semiconductors using a plane-wave basis set. Computational Materials Science. 1996;6(1):15-50.

[20] Kresse G, Furthmüller J. Efficient iterative schemes for ab initio total-energy calculations using a plane-wave basis set. Physical Review B. 1996;54(16):11169.

[21] Blöchl PE. Projector augmented-wave method. Physical Review B. 1994;50(24):17953.

[22] Perdew JP, Burke K, Ernzerhof M. Generalized Gradient Approximation Made Simple. Physical Review Letters. 1996;77(18):3865-8.

[23] Togo A, Tanaka I. First principles phonon calculations in materials science. Scripta Materialia. 2015;108:1-5.

[24] Gu X, Yang R. Phonon transport and thermal conductivity in two-dimensional materials. Annual Reviews of Heat Transfer. 2015;19:1-65.

[25] Esfarjani K, Chen G, Stokes HT. Heat transport in silicon from first-principles calculations. Physical Review B. 2011;84(8):085204.

[26] Li W, Lindsay L, Broido D, Stewart DA, Mingo N. Thermal conductivity of bulk and nanowire $\mathrm{Mg} 2 \mathrm{Si}$ x $\mathrm{Sn} 1-\mathrm{x}$ alloys from first principles. Physical Review B. 2012;86(17):174307. 
[27] $\mathrm{Gu} \mathrm{X}, \mathrm{Li}$ B, Yang R. Layer thickness-dependent phonon properties and thermal conductivity of MoS2. Journal of Applied Physics. 2016;119(8):085106.

[28] Gu X, Yang R. Phonon transport in single-layer transition metal dichalcogenides: A firstprinciples study. Applied Physics Letters. 2014;105(13):131903.

[29] Gu X, Yang R. First-principles prediction of phononic thermal conductivity of silicene: A comparison with graphene. Journal of Applied Physics. 2015;117(2):025102.

[30] Plimpton S. Fast parallel algorithms for short-range molecular dynamics. Journal of computational physics. 1995;117(1):1-19.

[31] Lindsay L, Broido D. Optimized Tersoff and Brenner empirical potential parameters for lattice dynamics and phonon thermal transport in carbon nanotubes and graphene. Physical Review B. 2010;81(20):205441.

[32] Kubo R, Toda M, Hashitsume N. Statistical physics II: nonequilibrium statistical mechanics: Springer Science \& Business Media; 2012.

[33] Hardy RJ. Energy-flux operator for a lattice. Physical Review. 1963;132(1):168.

[34] Merklein M, Lechler J, Geiger M. Characterisation of the flow properties of the quenchenable ultra high strength steel 22MnB5. CIRP Annals-Manufacturing Technology. 2006;55(1):229-32.

[35] Cheng S, Spencer J, Milligan W. Strength and tension/compression asymmetry in nanostructured and ultrafine-grain metals. Acta Materialia. 2003;51(15):4505-18.

[36] Xin Z, Jianjun Z, Zhong-Can O-Y. Strain energy and Young's modulus of single-wall carbon nanotubes calculated from electronic energy-band theory. Physical Review B. 2000;62(20):13692. 
[37] Vodenitcharova T, Zhang L. Effective wall thickness of a single-walled carbon nanotube. Physical Review B. 2003;68(16):165401.

[38] Yu M-F, Files BS, Arepalli S, Ruoff RS. Tensile loading of ropes of single wall carbon nanotubes and their mechanical properties. Physical review letters. 2000;84(24):5552.

[39] Baskin Y, Meyer L. Lattice constants of graphite at low temperatures. Physical Review. 1955;100(2):544.

[40] Wang B, Wu J, Gu X, Yin H, Wei Y, Yang R, et al. Stable planar single-layer hexagonal silicene under tensile strain and its anomalous Poisson's ratio. Applied Physics Letters. 2014;104(8):081902.

[41] Holzapfel GA. Nonlinear solid mechanics: Wiley Chichester; 2000.

[42] Toupin RA. Saint-Venant's principle. Archive for Rational Mechanics and Analysis. 1965;18(2):83-96.

[43] Gibson LJ, Ashby MF. Cellular solids: structure and properties: Cambridge university press; 1999.

[44] Haskins J, Kınacı A, Sevik C, Sevinçli H1, Cuniberti G, Çağın T. Control of thermal and electronic transport in defect-engineered graphene nanoribbons. ACS nano. 2011;5(5):377987.

[45] Evans WJ, Hu L, Keblinski P. Thermal conductivity of graphene ribbons from equilibrium molecular dynamics: Effect of ribbon width, edge roughness, and hydrogen termination. Applied Physics Letters. 2010;96(20):203112.

[46] Ye Z-Q, Cao B-Y, Yao W-J, Feng T, Ruan X. Spectral phonon thermal properties in graphene nanoribbons. Carbon. 2015;93:915-23. 
[47] Wang Y, Qiu B, Ruan X. Edge effect on thermal transport in graphene nanoribbons: A phonon localization mechanism beyond edge roughness scattering. Applied Physics Letters. 2012;101(1):013101. 